Research Paper

\title{
The Differential Expression and Function of the Inflammatory Chemokine Receptor CXCR5 in Benign Prostatic Hyperplasia and Prostate Cancer
}

\author{
Lu Yang*, Liang Gao*, Yongji Chen, Zhuang Tang, Yuchun Zhu, Ping Han, Xiang Li, Qiang Wei ${ }^{凶}$ \\ Department of Urology, West China Hospital, Sichuan University, No. 37 Guoxue Alley, Chengdu, Sichuan 610041, PR China. \\ * Lu Yang and Liang Gao contributed equally to this work and should share the co-first author. \\ $\square$ Corresponding author: Qiang Wei, MD., Professor and Chairman, Department of Urology, West China Hospital, Sichuan University, No. 37 \\ Guoxue Alley, Chengdu, Sichuan 610041, PR China. Phone and Fax: 86-28-85422451; E-mail: weiqiang163163@163.com
}

(c) 2015 Ivyspring International Publisher. Reproduction is permitted for personal, noncommercial use, provided that the article is in whole, unmodified, and properly cited. See http://ivyspring.com/terms for terms and conditions.

Received: 2015.01.27; Accepted: 2015.06.09; Published: 2015.10.15

\begin{abstract}
Background: Chemokine and chemokine receptors could have played an important role in tumor angiogenesis and distant metastasis. The mechanism of inflammation, expression and function of chemokines and chemokine receptors in benign prostatic hyperplasia (BPH) and prostate cancer $(\mathrm{PCa})$ remain unclear. The purpose of present study is to detect differential expression and function of chemokines and chemokine receptors (CCRs) in BPH and PCa.

Methods: BPH-1 and peripheral blood mononuclear cells (PBMCs) were co-cultured in Transwell chambers, and human normal prostate (NP) tissues, BPH tissues and PCa tissues were collected. CCR gene-chips were used to analyze and compare the differential expression of CCRs in BPH-1 cells, BPH-1 cells co-cultured with PBMCs, and LNCaP cells. The differential expression of CCRs was detected and validated using real-time PCR, western blotting and immunofluorescence (IF). The proliferation of LNCaP cells was also investigated after the knockdown CXCR5.

Results: Results of gene-chips indicated that there was low or no expression of CCR 10, CXCR 1, CXCR3 and CXCR5 in BPH-1 cells, whereas the expression of these receptors in BPH-1 cells was increased by PBMCs, and the expression was high in LNCaP cells. Furthermore, real-time PCR and western blotting confirmed the above mentioned results. IF verified no or low expression of CXCR1, CXCR3 and CXCR5 in NP tissues, low or moderate expression in BPH and high expression in PCa. However, CCR 10 was not expressed at detectable levels in the three groups. The growth and proliferation of LNCaP cells was markedly inhibited after down-regulation of CXCR5.

Conclusions: PCa cells expressed high levels of CCR 10, CXCR 1, CXCR3 and CXCR5. Although $\mathrm{BPH}$ cells did not express these factors, their expression was up-regulated when $\mathrm{BPH}-1$ cells were incubated with inflammatory cells. Finally, down-regulation of CXCR5 inhibited the growth and proliferation of LNCaP cells.
\end{abstract}

Key words: Chemokine, CXCR5, Differential expression, Inflammation, Prostate cancer

\section{Introduction}

Prostate cancer (PCa) is the most common malignant solid tumor, and it has the highest morbidity among all male tumor patients in Europe and North America. It is also a leading cause of cancer-related deaths among males in western countries [1]. Several factors have been implicated in this process; however, the mechanisms of PCa tumorigenesis remain unclear [2]. Benign prostatic hyperplasia (BPH) is a common benign disease in aging males. The morbidity of $\mathrm{BPH}$ is also high worldwide. However, the mechanism for the occurrence and development of $\mathrm{BPH}$ is remains unknown [3]. 
The relationship among the progression of inflammation, BPH and PCa is also yet to be elucidated. Based on studies of tissues obtained from prostate biopsies, $99.3 \%$ of BPH tissue samples were accompanied by tissue inflammation, which was characterized mainly by mild, multifocal and peri-glandular inflammation. In addition, $88.5 \%$ of PCa tissue samples also had tissue inflammation, which presented as mild, focal and interstitial inflammation [4]. These results suggest that tissue inflammation has a close relationship with $\mathrm{BPH}$ and $\mathrm{PCa}$. Some studies reported that $\mathrm{BPH}$ often coexists with $\mathrm{PCa}$ and that precancerous lesion-atypical small acinar proliferation (ASAP) generally co-exists with $\mathrm{BPH}$. Some reports have hypothesized that $\mathrm{BPH}$ might result in $\mathrm{PCa}$ or it might be an intermediary stage during tumorigenesis [5], whereas others have suggested that both are completely distinct conditions [6]. However, there is no direct evidence available to support either of the opinions. Therefore, a comprehensive study is required to explore the relationship and the mechanism of the progression of inflammation, in $\mathrm{BPH}$ and $\mathrm{PCa}$.

Chemokines are small cytokines produced by various cells, including white blood cells, fibroblasts and endothelial cells. These induce target cells, such as inflammatory cells, immune cells and stem cells, to undergo chemotactic movements. Chemokines are not only involved in the inflammatory response but also may participate in cancer tumorigenesis, progression and metastasis [7-9]. Specific combinations of chemokine receptors may play an important role in tumor behavior. For example, CXCL13/CXCR5 is the key signaling pathway in pyloric helicobacterium-related gastritis-induced ectopic mucosa-associated lymphoid tissue neoplasia [10]. CXCR4 is often highly expressed in various tumors, including breast, lung, prostate, stomach and pancreatic cancer [11]. Stromal-derived factor-1 (SDF-1) /CXCR4 signaling plays a critical role in tumor angiogenesis and distant metastasis [12]. However, the expression and function of chemokines and chemokine receptors in $\mathrm{BPH}$ and PCa is unclear. Therefore, in this study, the aim of this study was to explore the relationship and mechanism by which inflammation and inflammation-related chemokines regulate the development and progression of $\mathrm{BPH}$ and PCa.

\section{Materials and Methods}

\section{Cell culture and co-culture system}

The stable human androgen-dependent cell line LNCaP (ATCC\#CRL-1740) was obtained from ATCC (Manassas, VA, USA). Epithelial benign prostatic hyperplasia (BPH-1) cells were obtained from the Chinese Academy of Sciences (Shanghai, China). Periph- eral blood mononuclear cells (PBMCs), including lymphocytes, monocytes, natural killer (NK) cells and dendritic cells (DC), were obtained and separated from the blood of healthy volunteers $(50 \mathrm{ml})$ as needed. Here PBMCs were selected to be co-cultured with prostatic cells because it offers similar subtypes of inflammatory cells and could be one of the most appropriate experimental condition available to reproduce an inflammatory microenvironment in vitro. All cells were cultured in RPMI-1640 medium (Invitrogen, CA, USA) supplemented with $10 \%$ fetal bovine serum (FBS), $2 \mathrm{mmol} / 1$ glutamine, $50 \mathrm{U} / \mathrm{ml}$ penicillin and $50 \mu \mathrm{g} / \mathrm{ml}$ streptomycin. All cells were maintained in a humidified atmosphere containing $5 \% \mathrm{CO}_{2}$ at $37^{\circ} \mathrm{C}$.

Co-culture studies were performed using Transwell chambers (Corning), containing thousands of $1 \mu \mathrm{m}$ micropores. Six Transwell chambers were placed in 6-well plates. RPMI-1640 culture media was added to the interior of the bottom chamber and top chamber of the inserts and was allowed to hydrate for $2 \mathrm{~h}$ at $37^{\circ} \mathrm{C}$ with $5 \% \mathrm{CO}_{2}$. BPH-1 cells $\left(0.5-1 \times 10^{5}\right.$ cells $/ \mathrm{ml}$ ) were then added to the upper chambers. Simultaneously, PBMCs $\left(0.5-1 \times 10^{6}\right.$ cells $/ \mathrm{ml}$; a $10: 1$ ratio of PBMCs:BPH-1 cells) were added to the lower chambers. The BPH-1 and PBMCs were co-cultured for 7 days, and the BPH-1 cells were then harvested for further studies.

\section{Prostate specimens}

A total of 10 normal prostate (NP) tissue samples were obtained from patients undergoing radical cystectomy of bladder tumors ( $<50$ years old). In addition, $10 \mathrm{BPH}$ tissue samples, consisting of four cases of TURP and six radical cystectomy specimens ( $>50$ years old), and 10 prostate cancer tissue samples were obtained by radical prostatectomy. All samples were collected at West China Hospital of Sichuan University. The patients underwent either open or laparoscopic prostatectomy. Written informed consent was obtained from all patients.

\section{Research reagents and antibodies}

The primer sequences used in the current study were as follows: CXCR1, 5'-CTGAGCCCCAAGTGG AACGAGACA-3' and 5'-GCACGGAACAGAAGCT TTATTAGGA-3'; CXCR3, 5'-ACACCTTCCTGCTCCA CCTA-3' and '5'-GTTCAGGTAGCGGTCAAAGC-3'; CXCR5, 5'-TCCCCTCCTCACTCCCTTCCCAT-3' and 5'-CCTGCGGTTCCATCTGAGTGACATC-3'; and CCR10, 5'-GGGGATGAAGAGGACGCATACT-3' and 5'-CCTGGACATCGGCCTTGT-3'. Antibodies against CXCR1, CXCR3 and CXCR5 were purchased from Abcam Inc. Antibodies against CCR10 were acquired from Santa Cruz. The synthetic small interfering 
RNAs (siRNAs) targeting human CXCR5 were obtained from Stealth Select RNAi (Invitrogen) and HP Validated siRNA (Qiagen). PCR Arrays (SABio96; SuperArray Inc.) were purchased from Kangchen Genechip, Shanghai, China.

\section{Gene expression analysis using a chemo- kine-related PCR gene microarray}

The differential expression of chemokine-related genes was analysed using a PCR array. Briefly, RNA from BPH-1, co-cultured PBMC/BPH-1 and LNCaP cells was extracted according to standard protocols and converted to first strand cDNA using the RT2 First Strand Kit. The template was added to an instrument-specific, ready-to-use RT2 SYBR Green QPCR Master Mix. The resulting mixture was added to the wells $(25 \mu \mathrm{l} /$ well $)$ of a PCR array plate that had been pre-loaded with gene-specific primer sets $(25 \mu \mathrm{l}$ for 96-well plates); PCR was then performed. The threshold cycle $(\mathrm{Ct})$ values for all the genes on each PCR array were calculated using instrument-specific software, and the fold-changes in gene expression for pair-wise comparisons were calculated using the $\Delta \Delta \mathrm{Ct}$ method.

\section{Quantitative Real-time PCR (qRT-PCR)}

RNA was extracted from BPH-1, co-cultured PBMC/BPH-1 and LNCaP cells using TRIzol (Invitrogen), and then, cleaned using an RNeasy MinElute clean-up kit (Qiagen, Valencia, CA, USA). RNA was purified using RNeasy (Qiagen) or miRVANA (Ambion) kits with DNase digestion on RNeasy columns. Complementary DNA (cDNA) was generated using the High-Capacity cDNA Reverse Transcription Kit (Roche). Quantitative analysis of the change in gene expression was calculated using a real-time PCR machine (iQ5, Bio-Rad). The PCR cycling conditions were $94^{\circ} \mathrm{C}$ for $3 \mathrm{~min}$, followed by 40 cycles of $94^{\circ} \mathrm{C}$ for $15 \mathrm{~s}, 60^{\circ} \mathrm{C}$ for $20 \mathrm{~s}$ and $72^{\circ} \mathrm{C}$ for $40 \mathrm{~s}$. Real-time PCR was used to quantify mRNA expression. The reactions were performed in duplicate, and the value of $2-\Delta \Delta \mathrm{Ct}$ represented the relative level of target gene expression.

\section{Western blotting}

Western blotting was performed using total cell lysates, which were obtained by lysing cells in RIPA buffer (Sigma, St. Louis, MO). The protein concentration was then determined using a Bio-Rad assay. Proteins were separated on SDS-PAGE gels, transferred to PVDF membranes (Millipore), immunoblotted with antibodies, and visualized using an enhanced chemiluminescence detection system (Amersham Biosciences). The protein bands were quantified by densitometry using ImageJ gel analysis software (http://rsbweb.nih.gov/ij/). All values were nor- malized to $\beta$-actin.

\section{Immunofluorescence (IF) microscopy}

Samples of normal prostate, $\mathrm{BPH}$ and prostate cancer tissue were fixed in $10 \%$ formalin, embedded in paraffin, sectioned, and stained for CXCR1, CXCR3, CXCR5, CCR10 and glucagon using immunohistochemistry. The tissue sections were incubated with primary antibodies against CXCR1 (1:50, Abcam), CXCR3 (1:50, Abcam), CXCR5 (1:100, Abcam), and CCR10 (1:100, Santa Cruz Biotechnology) overnight at $4^{\circ} \mathrm{C}$, followed by FITC-conjugated secondary antibodies (1:250) for $1 \mathrm{~h}$ at room temperature. Blocking goat serum $(5 \%)$, primary and secondary antibodies were applied in PBS containing 0.3\% Triton X-100. The tissues slices were visualized using fluorescence microscopy, and the images were analysed using Advanced Sport software (Diagnostic Instruments, Sterling Heights, MI, USA).

\section{siRNA and transfection}

LNCaP cells were transfected with $100 \mathrm{nmol} / 1$ human CXCR5 or control siRNA (Santa Cruz) using with Lipofectamine 2000 according to the manufacturer's instructions. The media were removed $24 \mathrm{~h}$ after transfection, and the cells were then incubated in media containing 10\% FBS for another $24 \mathrm{~h}$. The efficiency of CXCR5 silencing was evaluated using real-time PCR and western blotting.

\section{Assessing cell proliferation using cell counting kit-8 (CCK8) and 5-bromo-2-deoxyuridine (BrdU)}

Cells were plated in 96-well plates at a density of $6 \times 103$ cells/well. The cells were transfected 100 nmol/1 human CXCR5 siRNA or control siRNA using Lipofectamine 2000 for $6 \mathrm{~h}$, and cell proliferation was analysed using a CCK-8 assay according to the manufacturer's instructions. The absorbance at $490 \mathrm{~nm}$ was then obtained using an enzyme-linked immunosorbent assay plate reader. To stain proliferating cells, LNCaP cells were stained with a FITC-conjugated anti-BrdU antibody (Biolegend) as well as DAPI. At least 150 cells were counted per condition, and the percentage of BrdU-positive cells is shown.

\section{BrdU staining and flow cytometry}

BrdU (Roche Applied Sciences Indianapolis, IN, USA) was dissolved in warm saline at a concentration of $10 \mathrm{mg} / \mathrm{ml}$. A final concentration of $50 \mu \mathrm{M}$ BrdU (RiBoBio, Guangzhou, China) was then added to cells for $12 \mathrm{~h}$ before harvesting. The supernatants were removed, and the resulting cells were stained using a FITC BrdU Flow Kit (BD Biosciences San Jose, CA, USA) according to the manufacturer's instructions. The cells were then resuspended in $1 \mathrm{ml}$ of staining 
buffer (PBS, 3\% FBS, 0.09\% sodium azide), and analysed by flow cytometry using a FACSCalibur flow cytometer. The data were analysed using BD CellQuest analysis software (BD Biosciences, San Diego, CA, USA).

\section{Ethics}

The design and execution of this study was approved by the Hospital Ethics Committee, and written informed consent was obtained from every patient involved. Research carried out in this study is in compliance with the Helsinki Declaration.

\section{Statistical analysis}

Statistical analyses were performed using analysis of variance followed by Tukey's multiple comparison tests or Student's t-test using SPSS16.0 software. A $p$ value of $<0.05$ was considered to be significant.

\section{Results}

\section{The expression of chemokines and their re- ceptors in BPH-1, co-cultured BPH-1 and PBMCs and LNCaP cells}

Gene expression profiling is important for discovering and validating the differences among various prostatic cell lines. RNA was extracted from BPH-1, BPH-1 after co-culture with PBMCs and $\mathrm{LNCaP}$ cells. Gene microarrays demonstrated that the expression of four chemokine receptors (CCR10, CXCR1, CXCR3 and CXCR5) increased gradually in BPH-1, BPH-1 after co-culture with PBMCs and LNCaP cells (Table 1).
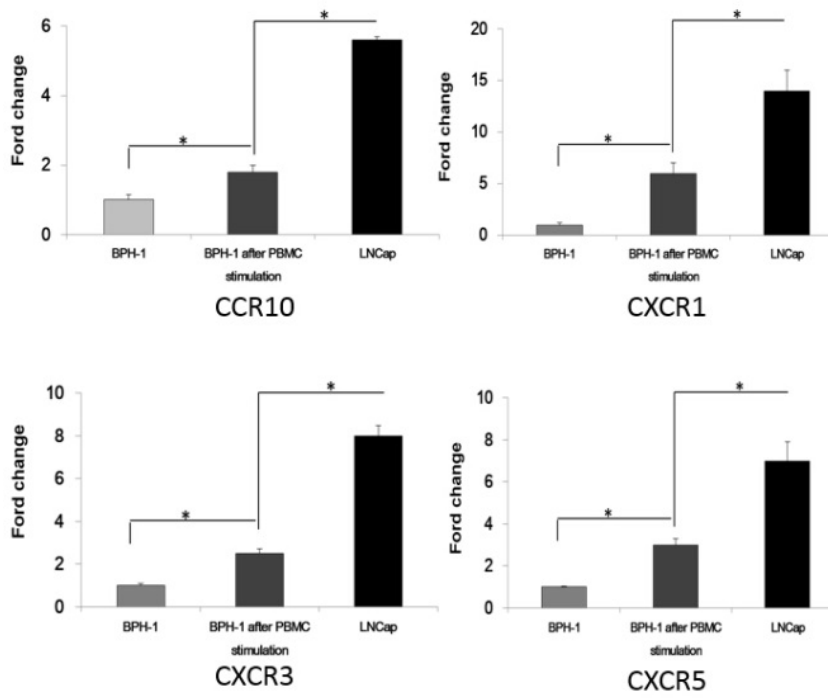

a
Table 1. More than 5-fold increase of chemokines and the receptors after inflammatory stimulation

\begin{tabular}{lll}
\hline Symbol & \multicolumn{2}{l}{ Fold Up- or Down- Regulation } \\
\cline { 2 - 3 } & BPH-1+PBMC/ BPH-1 & LNCaP / BPH-1+PBMC \\
\hline CCR10 & 6.33 & 6.36 \\
CXCR1 & 6.14 & 7.1 \\
CXCR3 & 5.69 & 8.06 \\
CXCR5 & 6.33 & 8.42 \\
\hline
\end{tabular}

PBMC: Peripheral blood mononuclear cell

In addition, qRT-PCR revealed that CCR10 mRNA was expressed in LNCaP cells at three-fold higher levels than in BPH-1 cells that were co-cultured with PBMCs, which was in turn 1.8-fold higher than in BPH-1 cells. CXCR1 mRNA in LNCaP cells was 2.4-fold higher than in BPH-1 cells co-cultured with PBMCs, which was 4.5-fold higher than in BPH-1 cells. CXCR3 mRNA in LNCaP cells was expressed at 3.6-fold higher levels than in BPH-1 cells co-cultured with PBMCs, which was in turn 2.3-fold higher than in $\mathrm{BPH}-1$ cells. Finally, CXCR5 mRNA as expressed at 2.4-fold higher levels in LNCaP cells than in BPH-1 cells co-cultured with PBMCs, which was three-fold higher than in BPH-1 cells (Figure 1).

Western blotting revealed that CCR10, CXCR1, CXCR3 and CXCR5 were either not expressed or were present at only very low levels in BPH-1 cells. After BPH-1 cells were co-cultured with PBMCs, CCR10, CXCR1, CXCR3 and CXCR5 were all up-regulated. CCR10, CXCR1, CXCR3 and CXCR5 were present at the highest levels in LNCaP cells (Figure 1).

\section{CCR10}

\section{CXCR1}

CXCR3

\section{CXCR5}

ACTIN

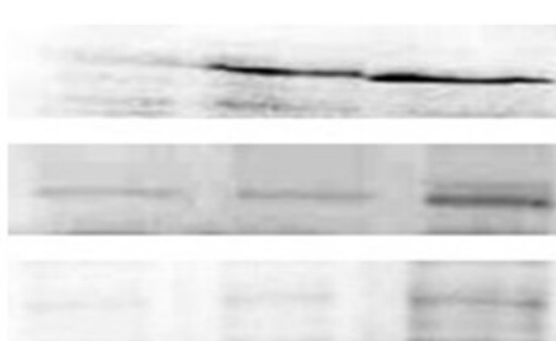

BPH-1 BPH-1+PBMC LNCaP

Figure 1 The expression of CCR10, CXCR1, CXCR3 and CXCR5. a) RT-PCR analysis of several cytokine receptors of three groups (BPH-1, BPH-1 after co-culture with PBMCs and LNCaP). Data represent three independent experiments performed in triplicate. Data were normalized to $\beta$-actin levels and are shown as normalized fold change when compared with BPH-1. *P < 0.05. b) Total cell lysates (60 ug) from cells were resolved on SDS-PAGE and subjected to immunoblotting using antibodies. $\beta$-actin was used as loading control. 


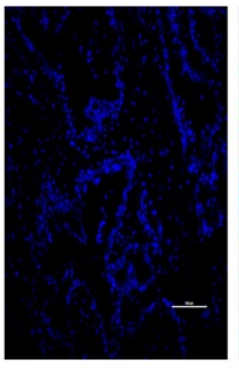

Normal prostate

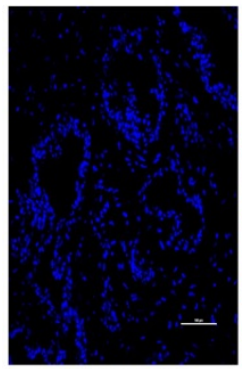

Normal prostate

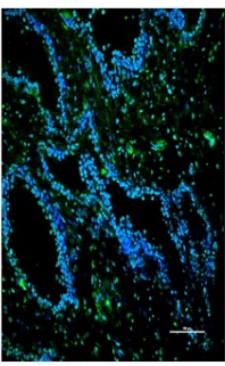

BPH
CXCR1

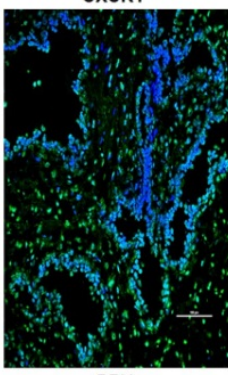

BPH
CXCR5

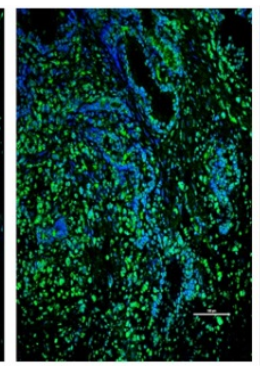

$\mathrm{PCa}$

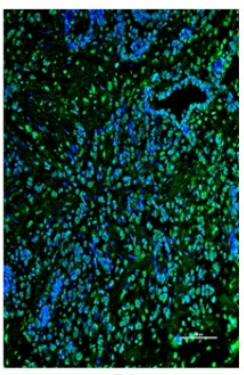

$\mathrm{PCa}$

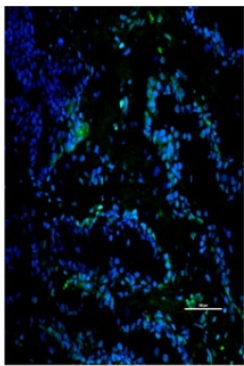

Normal prostate

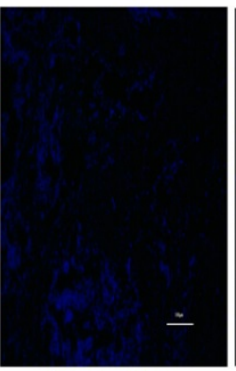

Normal prostate

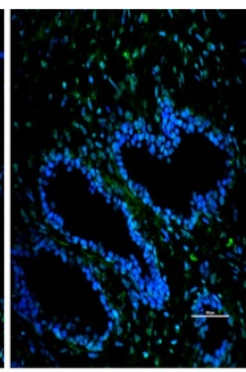

CXCR3

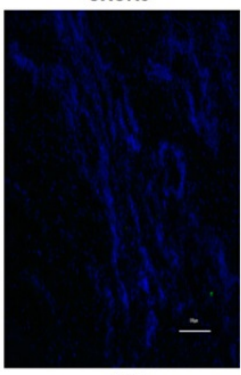

BPH

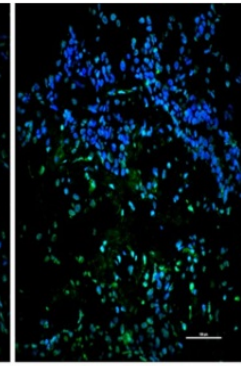

$\mathrm{PCa}$

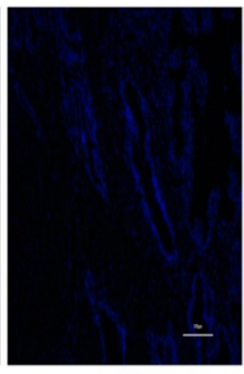

PCa

Figure 2 Representative immunofluorescence staining of CCR10, CXCR1, CXCR3 and CXCR5 on the tissular levels of NP, BPH and PCa. CCR10, CXCR1, CXCR3 and CXCR5 are shown by Alexa Fluor 488 (green) and nuclear staining with 4,6-diamidino-2-phenylindole (DAPI; blue). All the pictures are merged from different type of tissues. The Scale bar represents $100 \mu \mathrm{m}$.

\section{The expression of chemokines and their re- ceptors in NP, BPH and PCa tissues}

IF was used to verify the results observed in cell lines at the tissue level. CXCR1, CXCR3 and CXCR5 were either not detectable or were expressed at only low levels in NP tissue. Conversely, they were less or moderately expressed in BPH tissue and highly expressed in PCa. However, CCR10 was either not present or expressed at very low levels in all tissues (Figures 2 and 3).
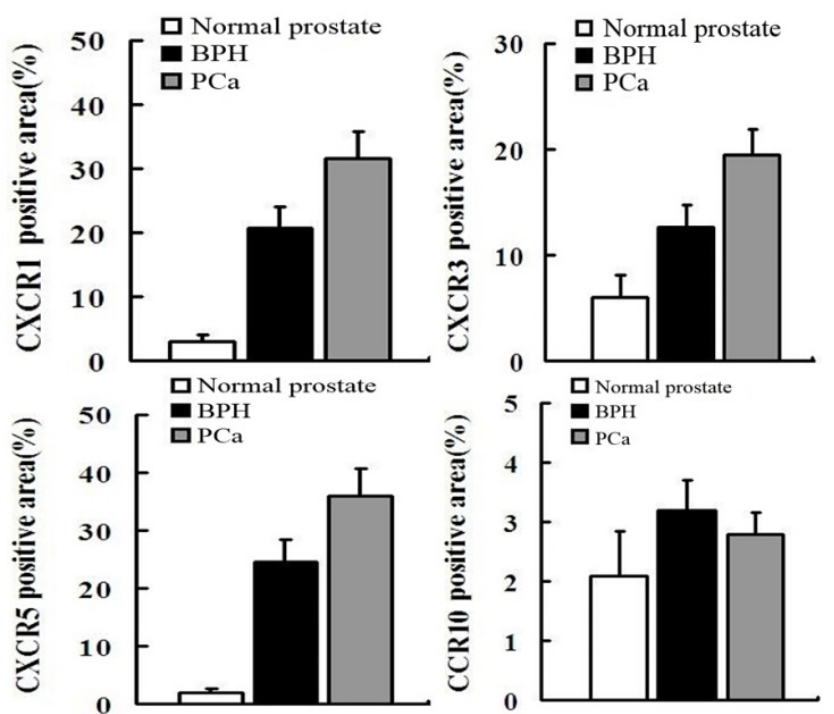

Figure 3 In order to quantify the differences between three groups (normal prostate, $\mathrm{BPH}, \mathrm{PCa}$ ), we select five field randomly in each slide. These images were analyzed using Advanced Sport software (Diagnostic Instruments, Sterling Heights, MI). Data show that CCR 10 was expressed at very low levels in all groups $(P>0.05)$. However, CXCR1, CXCR3 and CXCR5 were highly expressed in PCa tissues compare to normal prostate or BPH $(\mathrm{P}<0.01)$.

\section{The effect of knocking down CXCR5 in LNCaP cells using siRNA}

To explore the function of CXCR5 in PCa cells, we knocked down CXCR5 expression using CXCR5-specific siRNA. As shown in Figures 4 and 5, transfection with CXCR5-siRNA effectively inhibited CXCR5 mRNA and protein expression in LNCaP cells, as confirmed using qRT-PCR and western blotting, respectively. Compared with blank and control siRNA-transfected cells, LNCaP cells transfected with CXCR5-siRNA exhibited significantly decreased cell proliferation for 3 days $(\mathrm{P}<0.01)$, as measured using a CCK8 assay (Figure 6) and BrdU assay (Figure 7, Figure 8). Therefore, these in vitro results further confirm the putative oncogenic role of CXCR5, which promotes the proliferation of PCa cells.

\section{Discussion}

In 1886, Virchow observed that several different tumors originated from chronic inflammatory lesions, and hypothesized that a close relationship was present between inflammation and neoplasms. It was reported that $20 \%$ of human tumors were related to inflammation, including carcinomas of the esophagus, stomach, colon-rectum, liver and pancreas [12]. Studies showed that the morbidity of colorectal tumors was 5-7 times higher in patients with inflammatory bowel diseases (such as ulcerative colitis and Crohn's disease) compared with those without any disease [13]. Rothwell et al. conducted a study in a 
cohort with long-term aspirin use for the prevention of cardiovascular diseases, and found that the tumor risk was reduced by $60 \%$ in the gastrointestinal tract and $30 \%$ for other solid tumors over a 20 year period [14]. However, in the prostate, an organ that is often infiltrated with inflammation, it remains unclear whether inflammation has a similar effect on the pathogenesis of $\mathrm{PCa}$, and what role it plays.

Tissue inflammation in the prostate results in the formation of chronic lesions, which are infiltrated by lymphocytes, mononuclear cells and macrophages; sometimes a small number of plasmocytes and acidocytes are also present. Numerous studies, have analysed the relationship between inflammation and $\mathrm{PCa}$, and revealed that a large proportion of $\mathrm{PCa}$ cases are accompanied by inflammation, which suggests that inflammation is an important factor in the pathogenesis of PCa. For example, in the California Men's Health Study (CMHS), Cheng et al. observed that patients with chronic prostatitis or related symptoms had a higher risk of PCa (OR=1.3) [15]. In a study of 5821 older males, Daniels et al. found that the mor- bidity of PCa was 5.4-fold higher in patients with prostatitis compared with those without $(\mathrm{P}<0.0001)$, and also demonstrated that might play an important role in PCa tumorigenesis [16].

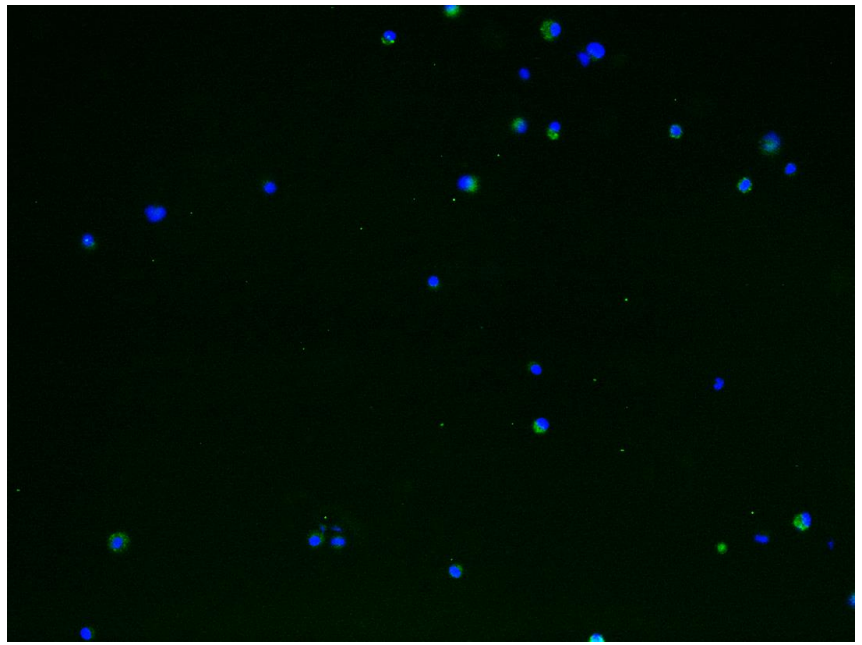

Figure 4 The transfection efficiency of LNCaP by siRNA-CXCR5 (More than $90 \%$ LNCaP cells were transfected). $100 \mathrm{nmol} / \mathrm{l}$ human CXCR5 or control siRNA (Santa Cruz) using with Lipofectamine 2000 according to the manufacturer's instructions.

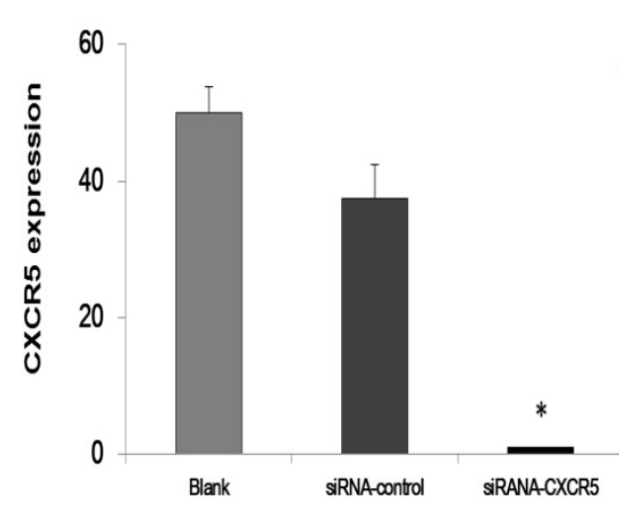

CXCR5

GAPDH

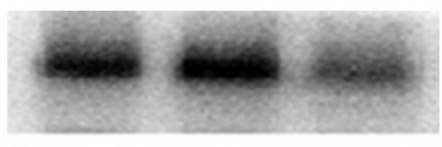

\section{GAPDH}
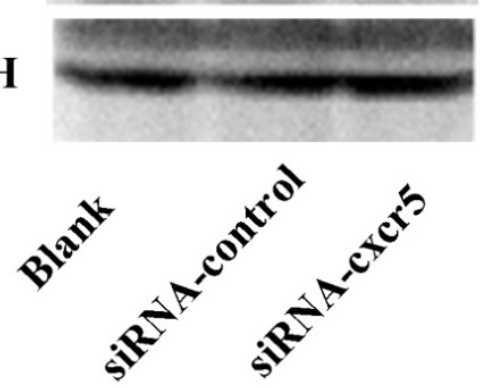

Figure 5 Western blot and RT-PCR analysis of CXCR5 protein levels in LNCaP cells transfected with scrambled siRNA (control siRNA) or siRNA targeting CXCR5. The results demonstrated the significant inhibition of CXCR5 mRNA and protein on LNCaP cells.

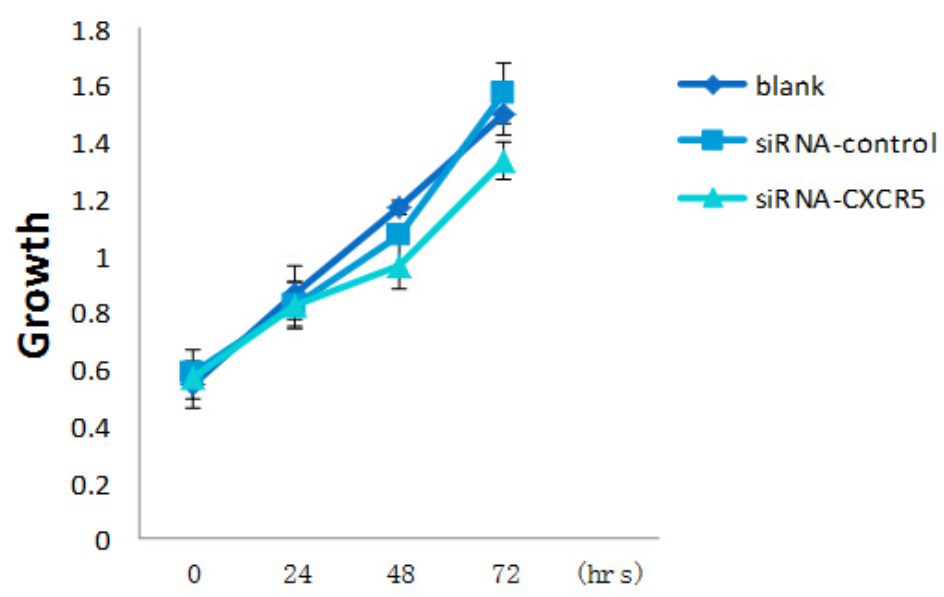

Figure $6 \mathrm{LNCaP}$ cells were grown in presence of CXCR5-siRNA or control siRNA or blank. CCK-8 assay was performed every $24 \mathrm{~h}$ for 3 days to assess cell proliferation. Error bars represent \pm standard error of means of three independent experiments. *Significant $(P<0.05)$ changes relative to blank cells. The CCK 8 showed the proliferative inhibition of $\mathrm{LNCaP}$ cells after CXCR5-siRNA transfection. 


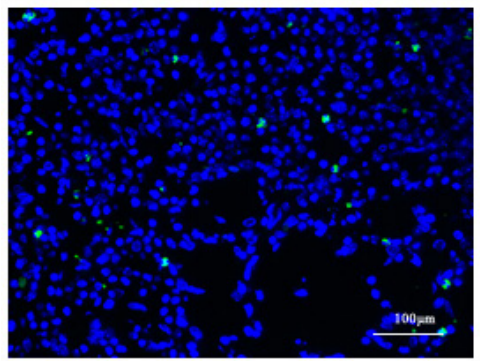

Blank

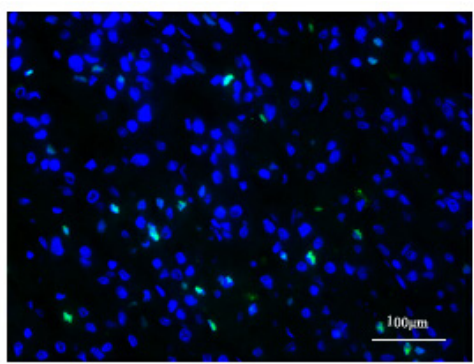

siRNA-control

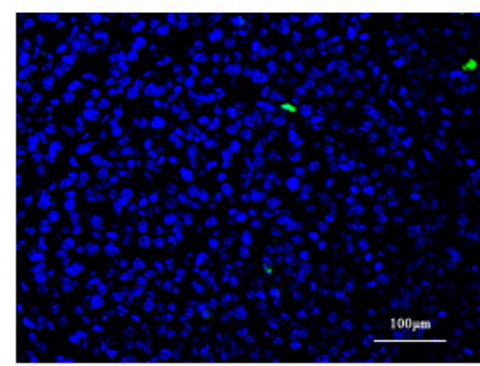

siRNA-CXCR5

Figure 7 The proliferative cell is shown by Alexa Fluor 488 (green). The BrdU showed the proliferative inhibition of LNCaP cells after CXCR5-siRNA transfection after 72 hrs.
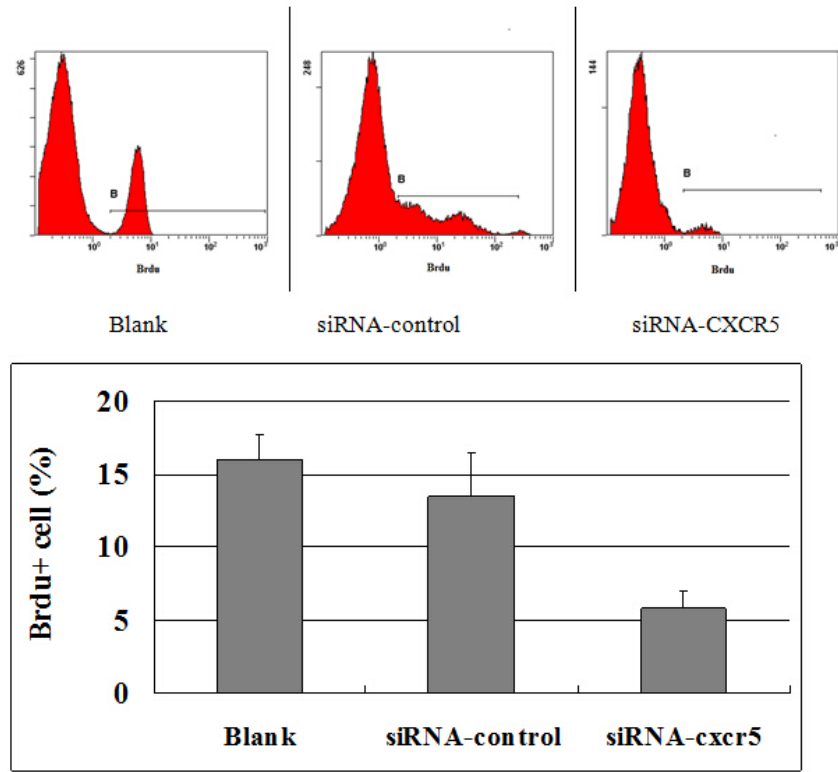

Figure 8 The FACSCalibur detected the positive cells of BrdU to reflect the proliferation of LNCaP cells was inhibited. The data were analysed using BD CellQuest analysis software (BD Biosciences, San Diego, CA, USA).

Subsequently, Gregory et al. reported the biopsy results of 177 cases of suspected PCa at the beginning but excluded later. They found that 144 biopsy tissues also exhibited chronic tissue inflammation whereas the remaining 33 cases did not. In a five-year follow-up, 29 cases with concurrent inflammation were diagnosed pathologically be PCa (approximately $20 \%)$, compared with two cases of PCa from the non-inflammatory cases (approximately $6 \%$ ). In addition, the morbidity of PCa patients with inflammation was significantly higher than those without inflammation [17].

The relationship between $\mathrm{BPH}$ and PCa is unclear. Although some scientists and clinicians consider BPH and PCa to be different diseases [6], others argue that there are some identical features to both $\mathrm{BPH}$ and $\mathrm{PCa}$; therefore, $\mathrm{BPH}$ might be an intermediary stage of $\mathrm{PCa}$ [5]. Inflammation is often present in either $\mathrm{BPH}$ or $\mathrm{PCa}$, and thus inflammation is assumed to play an essential role in the pathogenesis of $\mathrm{BPH}$ and PCa despite a lack of adequate evidence.

Chemokines and their receptors are character- ized by their involvement in inflammation. However, increasing evidence suggests that these factors also play a crucial role in neoplastic transformation, cancer cell migration, invasion and metastasis [18]. The chemokine receptor, CXCR5, is expressed primarily by $B$ cells and certain $T$ cells, and it regulates their migration into and within lymph nodes. CXCL13, the ligand of CXCR5, interacts with CXCR5 and activates chemotaxis [19].

Recently, some studies assessing the role of CXCR5 in tumors have emerged. CXCR5, which was isolated originally from Burkitt lymphoma, was recently detected in breast carcinoma tissues [20-22]. Del Grosso et al. demonstrated that CXCL13 and its receptor, CXCR5, are involved in the relationship between neuroblastic and Schwannian stromal cells in neuroblastic tumors [22]. Meijer et al. observed that CXCR5 was expressed in the colon carcinoma and that it mediated metastasis to the liver [23].

In $\mathrm{PCa}$, Singh et al. [24] detected the expression of CXCR5 in PCa cells, and reported that it contributed to tumor growth and invasion. El-Haibi et al. [25] 
also observed that CXCL13 could mediate prostate cancer cell proliferation via JNK signaling, invasion via ERK activation and also activate CXCR5. In a subsequent study, El-Haibi et al. [26] demonstrated that some therapeutic strategies involving CXCR5 and/or CXCL13 blockade and specific G protein inhibition were beneficial for abrogating PCa metastasis.

After a comparison of BPH with PCa in the current study, CXCR5 was identified and analysed further because of its simple relationship with its ligands, a lack of studies on its role in PCa and its future research potential [27]. Our observations were consistent with those of Singh et al. [24], whereby CXCR5 mRNA and protein expression was elevated significantly in PCa cell lines and PCa tissues. The current study also revealed that CXCR5 mRNA and protein were either not present or were expressed at extremely low levels in $\mathrm{BPH}$ cell lines. However, although the presence of inflammatory cells up-regulated their expression, their levels were still lower than in tumor cells. On the other hand, normal prostate tissue and $\mathrm{BPH}$ tissue did not highly express CXCR5. A functional assay revealed that knocking down CXCR5 in LNCaP cells inhibited tumor cell proliferation.

There are some limitations to the present study. First, the study assessing the function of CXCR5 was insufficient to clarify its role in tissue inflammation and PCa tumorigenesis. Furthermore, this study was mainly used in vitro approaches and thus additional in vivo studies are needed. Finally, this study focused on CXCR5, and hence the ligand, CXCL13, and the other chemokine receptors identified should be investigated in the future.

\section{Conclusions}

PCa cells expressed high level of CCR10, CXCR1, CXCR3 and CXCR5, whereas BPH cells either did not express the abovementioned chemokine receptors or they were present at only very low levels. However, their expression was up-regulated in the presence of inflammatory cells. Down-regulating the expression of CXCR5 inhibited the growth and proliferation of androgen-dependent PCa cells. Chemokines and their receptors, particularly CXCR5, might participate in the development of prostate tissue inflammation and the tumorigenesis of prostate cancer cells.

\section{Abbreviations}

ASAP: atypical small acinar proliferation; $\mathrm{BPH}$ : benign prostatic hyperplasia; BrdU: 5-bromo-2-deoxyuridine; CCK8: cell counting kit-8; CCRs: chemokines and chemokine receptors; cDNA: complementary DNA; CMHS: California Men's
Health Study; DC: dendritic cells; FBS: fetal bovine serum; IF: immunofluorescence; NK: natural killer; NP: normal prostate; PBMCs: peripheral blood mononuclear cells; PCa: prostate cancer; SDF-1: stromal-derived factor-1; siRNAs: small interfering RNAs.

\section{Acknowledgement}

This study was supported by the Prostate Cancer Foundation Young Investigator Award 2013, the National Natural Science Foundation of China (Grant No. 81300627, 81370855 and 30901484) and Programs from Science and Technology Department of Sichuan Province (Grant No. $2013 S Z 0006$ and 2014JY0219).

\section{Competing Interests}

The authors have declared that no competing interest exists.

\section{References}

1. Siegel R, Naishadham D, Jemal A. Cancer Statistics, 2013. CA-Cancer J Clin. 2013; 63: 11-30.

2. Wangerin H, Kristiansen G, Schlomm T, et al. CD57 expression in incidental, clinically manifest, and metastatic carcinoma of the prostate. Biomed Res Int. 2014;: 356427.

3. Parsons JK. Benign prostatic hyperplasia and male lower urinary tract symptoms: epidemiology and risk factors. Curr Bladder Dysfunct Rep. 2010; 5: 212-218.

4. Yang L, Zhao K, Zhang A, et al. Differences in pathological features of histological inflammation between benign prostatic hyperplasia and prostate cancer. Journal of Sichuan University (medicine). 2013; 44: 760-763.

5. Alcaraz A, Hammerer P, Tubaro A, et al. Is there evidence of a relationship between benign prostatic hyperplasia and prostate cancer? findings of a literature review. Eur Urol. 2009; 55: 864-875.

6. Bostwick DG, Burke HB, Djakiew D, et al. Human prostate cancer risk factors. Cancer. 2004; 101(Suppl 10): 2371-2490.

7. Balkwill F. Cancer and the chemokine network. Nat Rev Cancer. 2004; 4: 540-550.

8. Mantovani A, Savino B, Locati M, et al. The chemokine system in cancer biology and therapy. Cytokine Growth F R. 2010; 21: 27-39.

9. Slettenaar VI, Wilson JL. The chemokine network: a target in cancer biology? Adv Drug Deliver Rev. 2006; 58: 962-974.

10. Winter S, Loddenkemper $\mathrm{C}$, Aebischer A, et al. The chemokine receptor CXCR5 is pivotal for ectopic mucosa-associated lymphoid tissue neogenesis in chronic Helicobacter pylori-induced inflammation. J Mol Med. 2010; 88: 1169-1180.

11. Sun YX, Wang J, Shelburne CE, et al. Expression of CXCR4 and CXCL12 (SDF-1) in human prostate cancers (PCa) in vivo. J Cell Biochem. 2003; 89: $462-473$.

12. Omab M, Ezeani M. Infection, inflammation and prostate carcinogenesis. Infect Genet Evol. 2011; 11: 1195-1198.

13. Ferrone C, Dranoff G. Dual roles for immunity in gastrointestinal cancers. J Clin Oncol. 2010; 28: 4045-4051.

14. Rothwell PM, Fowkes FG, Belch JF, et al. Effect of daily aspirin on long-term risk of death due to cancer: analysis of individual patient data from randomized trials. Lancet. 2011; 377: 31-41.

15. Cheng I, Witte JS, Jacobsen SJ, et al. Prostatitis, sexually transmitted diseases, and prostate cancer: the California Men's Health Study. PLoS ONE. 2010; 5: e8736.

16. Daniels NA, Ewing SK, Zmuda JM, et al. Correlates and prevalence of prostatitis in a large community-based cohort of older men. Urology. 2005; 66: 964-970.

17. Gregory TM, Rosana E, Ranleigh LF, et al. The influence of chronic inflammation in prostatic carcinogenesis: a 5-year follow-up study. J Urol. 2006; 176: 1012-1016.

18. Keeley EC, Mehrad B, Strieter RM. CXC chemokines in cancer angiogenesis and metastases. Adv Cancer Res. 2010; 106: 91-111.

19. Hussain SK, Zhu W, Chang SC, et al. Serum levels of the chemokine CXCL13, genetic variation in CXCL13 and its receptor CXCR5, and HIV associated non-hodgkin B-cell lymphoma risk. Cancer Epidem Biomar. 2013; 22: 295-307.

20. Panse J, Friedrichs K, Marx A, et al. Chemokine CXCL13 is overexpressed in the tumor tissue and in the peripheral blood of breast cancer patients. Brit J Cancer. 2008; 99: 930-938. 
21. Razmkhah M, Jaberipour M, Safaei A, et al. Chemokine and chemokine receptors: a comparative study between metastatic and non-metastatic lymph nodes in breast cancer patients. Eur Cytokine Netw. 2012; 23: 72-77.

22. Del Grosso F, Coco S, Scaruffi P, et al. Role of CXCL13-CXCR5 crosstalk between malignant neuroblastoma cells and Schwannian stromal cells in neuroblastic tumors. Mol Cancer Res. 2011; 9: 815-823.

23. Meijer I, Zeelenberg IS, Sipos B, et al. The CXCR5 chemokine receptor is expressed by carcinoma cells and promotes growth of colon carcinoma in the liver. Cancer Res. 2006; 66: 9576-9582.

24. Singh S, Singh R, Singh UP, et al. Clinical and biological significance of CXCR5 expressed by prostate cancer specimens and cell lines. Int J Cancer. 2009; 125: 2288-2295.

25. El-Haibi CP, Singh R, Sharma PK, et al. CXCL13 mediates prostate cancer cell proliferation through JNK signalling and invasion through ERK activation. Cell Proliferat. 2011; 44: 311-319.

26. El-Haibi CP, Sharma P, Singh R, et al. Differential G protein subunit expression by prostate cancer cells and their interaction with CXCR5. Mol Cancer. 2013; 12: 64 .

27. Zhang L, Yang BX, Zhang HT, et al. Prostate cancer: an emerging threat to the health of aging men in Asia. Asian J Androl. 2011; 13: 574-578 